\title{
Effects on CD3, Treg, and TH17 Cell Numbers in Skin Biopsies after 16-Week Mirikizumab Treatment, Evaluated by an Epigenetic Assay
}

\section{Robert Bissonnette1, Jochen Schmitz², Dipak Patel${ }^{2}$, Richard E. Higgs ${ }^{2}$, Henrik H. Sonnergren², Karen Huayu Liu ${ }^{2}$, Kristian Reich, ${ }^{3}$ Elisabeth RiedI ${ }^{*}$}

1Innovaderm Research, Montreal, Quebec, Canada; ${ }^{2}$ Eli Lilly and Company, Indianapolis, IN, USA; 3Professorship for Translational Research in Inflammatory Skin Diseases, Institute for Health Services Research in Dermatology and Nursing, University Clinic Hamburg-Eppendorf, and Skinflammation ${ }^{\circledR}$ Center, Hamburg, Germany; ${ }^{4}$ Eli Lilly Regional Operations GmbH, Vienna, Austria *Presenter only

\section{INTRODUCTION}

- Study I6T-MC-AMAF is a double-blind phase 2 trial to determine safety and efficacy of LY3074828 (mirikizumab), a humanized monoclonal antibody against the p19 subunit of

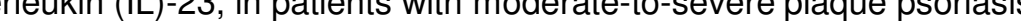

IL-23 is a proinflammatory cytokine produced primarily by myeloid cells It drives the secretion of IL-17 from T cells and other innate immune cells ${ }^{1-3}$

- Mirikizumab treatment led to a significant improvement in clinical response compared to placebo at Week 16 (66.7\% of patients in $300 \mathrm{mg}$ group achieved Psoriasis Area and Severity Index PASI]90) ${ }^{4}$

- Past studies showed that even when psoriasis is clinically resolved, IL-17-producing cells can remain in skin after treatment

Their presence may potentially contribute to flares after anti-IL-17 withdrawa

To determine the cellular skin milieu of patients in the current study, we evaluated the relationships between mirikizumab exposure, efficacy, and skin biomarkers

\section{HYPOTHESIS}

By blocking IL-23p19, mirikizumab decreases the IL-17-producing cells within the skin, reducing disease severity and the potential for flares

\section{STUDY DESIGN}

\section{Mirikizumab AMAF Phase 2 Trial Design}

- Study I6T-MC-AMAF is a Phase 2, multicenter, randomized, parallel-arm, placebo-controlled study of mirikizumab in subjects with moderate-to-severe plaque psoriasis (NCT02899988)

- Patients with chronic plaque psoriasis (body surface area $\geq 10 \%$, PASI $\geq 12$, static Physician's Global Assessment $\geq 3$ ) received mirikizumab 300,100 , or $30 \mathrm{mg}$ subcutaneously or placebo every 8 weeks for 16 weeks

- Lesional skin biopsies from each group were assessed at baseline and Week 16 using the EpiontisIDTM assay: cluster of differentiation (CD)3, regulatory T cell (Treg), IL-17-producing cells

- Post hoc analyses: (1) Fold change in cell-type frequency over baseline; (2) Correlation coefficient between cell-type frequency and Week 16 PASI changes

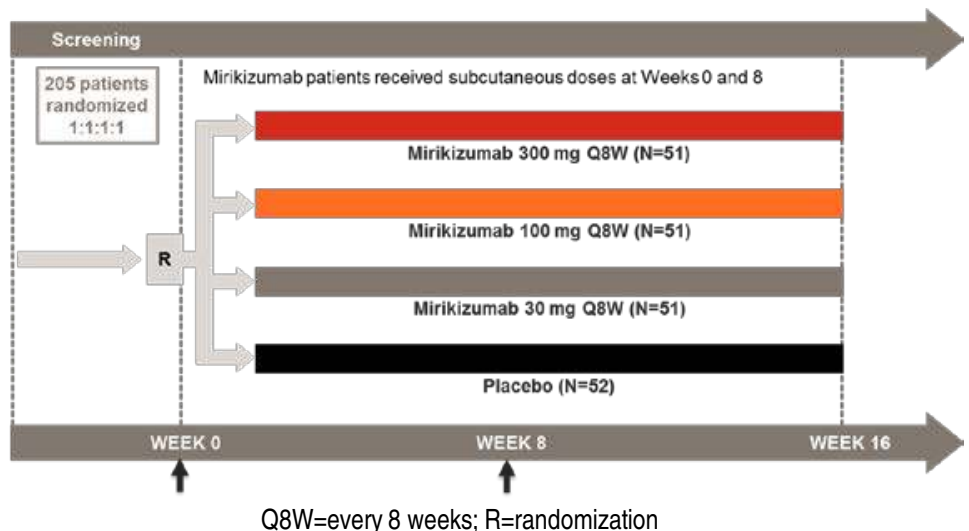

\section{KEY RESULT}

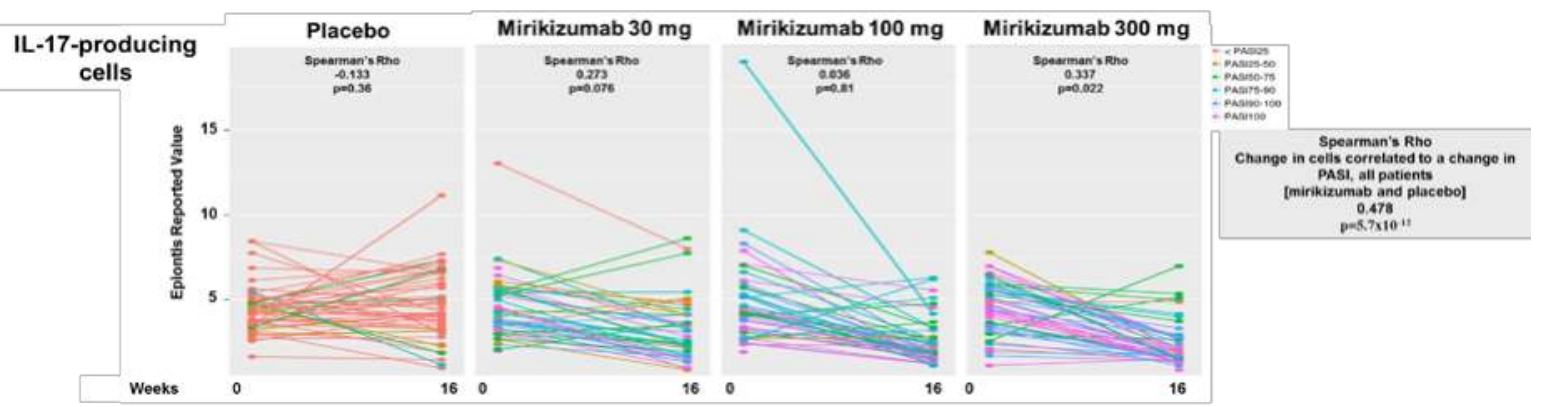

Reductions in IL-17-producing cells were associated with improvements in PASI

\begin{tabular}{|c|c|c|c|c|c|}
\hline \multirow{2}{*}{$\begin{array}{l}\text { Other } \\
\text { Cell } \\
\text { Types }\end{array}$} & \multicolumn{5}{|c|}{ Correlation Coefficient: Spearman's Rho, $p$-Value Change in cells vs PASI } \\
\hline & Placebo & $\begin{array}{l}\text { Mirikizumab } \\
30 \mathrm{mg}\end{array}$ & $\begin{array}{l}\text { Mirikizumabo } \\
100 \mathrm{mg}\end{array}$ & $\begin{array}{l}\text { Mirikizumab } 300 \\
\text { mg }\end{array}$ & $\begin{array}{l}\text { All Mirikizumab } \\
\text { Doses }\end{array}$ \\
\hline CD3 & $\begin{array}{l}-0.123 \\
p=0.41\end{array}$ & $\begin{array}{c}0.325 \\
p=0.029\end{array}$ & $\begin{array}{l}-0.017 \\
p=0.91\end{array}$ & $\begin{array}{c}0.091 \\
p=0.54\end{array}$ & $\begin{array}{c}0.399 \\
p=1.7 \times 10^{-8}\end{array}$ \\
\hline Treg & $\begin{array}{l}-0.149 \\
\mathrm{p}=0.31\end{array}$ & $\begin{array}{c}0.432 \\
p=0.0031\end{array}$ & $\begin{array}{l}0.297 \\
p=0.05\end{array}$ & $\begin{array}{l}0.202 \\
p=0.17\end{array}$ & $\begin{array}{c}0.489 \\
\mathrm{p}=1.8 \times 10^{-12}\end{array}$ \\
\hline
\end{tabular}

$\mathrm{CD}=$ =luster of differentiation; IL=interleukin; PASI=Psoriasis Area and Severity Index; Treg=regulatory T cell

\section{EpiontisIDTM Assay}

Used to identify cells within skin biopsies via cell type-specific epigenetic biomarkers in CpGdemethylated regions of DNA

$\mathrm{CpG}$ demethylation is an indicator of a cell's digital phenotype

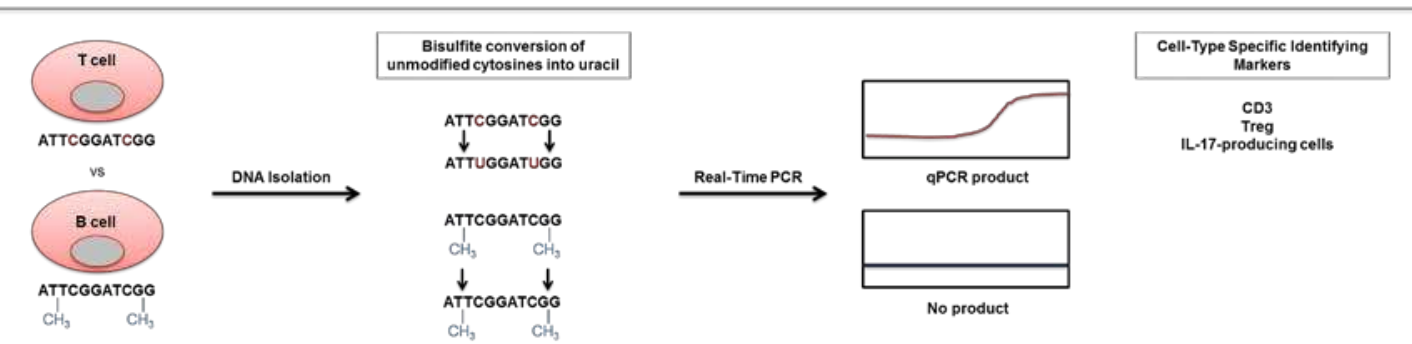

\section{Advantages}

- Most clinical biopsies are limited to the detection of cell subsets either by immunohistochemistry HC), gene expression analysis, or flow cytometry:

IHC and gene expression analysis have significant problems in terms of true cell counting; both methods are limited in formal quantification of cell numbers

While flow cytometry allows for accurate cell enumeration, the need of fresh biopsies generates logistical challenges that would only allow a very limited subset of patients to be assessed

The epigenetic cell counting methodology allows the assessment of the whole studied AMAF patient population $\rightarrow 205$ patients with biopsies from 2 time points
AMAF Study Outcomes at Week 16

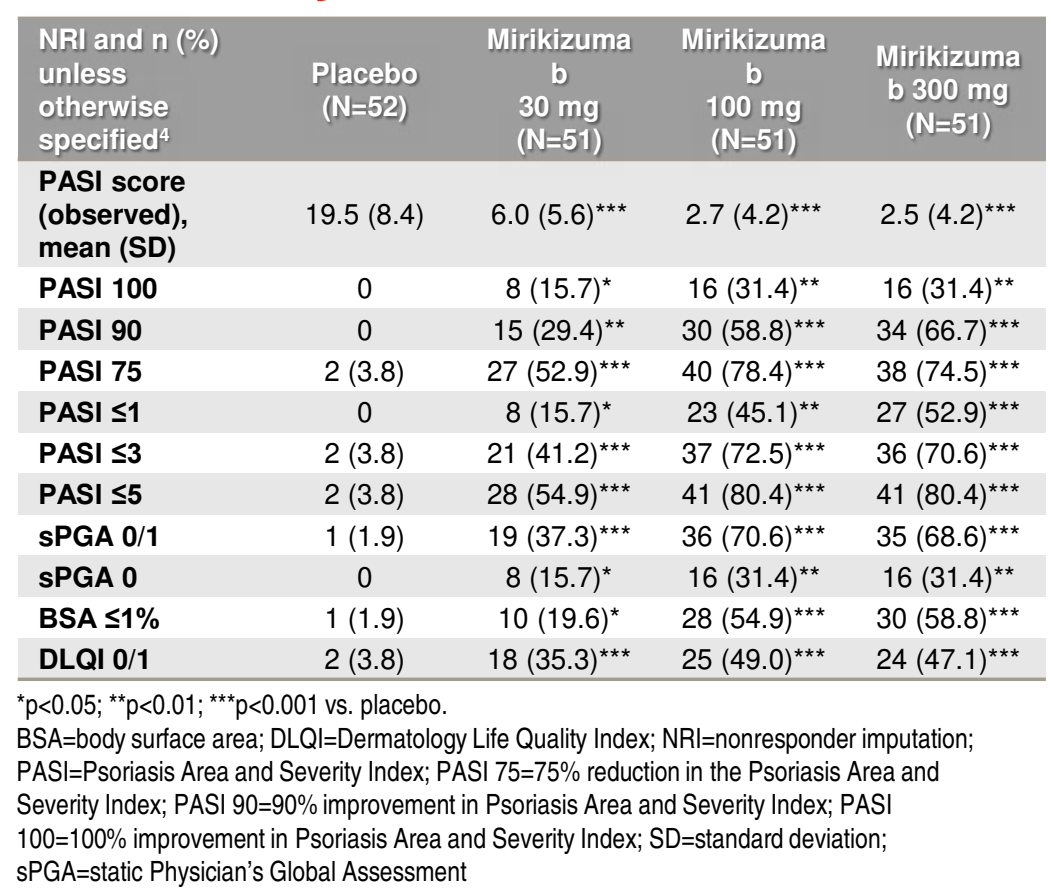

Distribution of Cell Populations Within Skin Biopsies

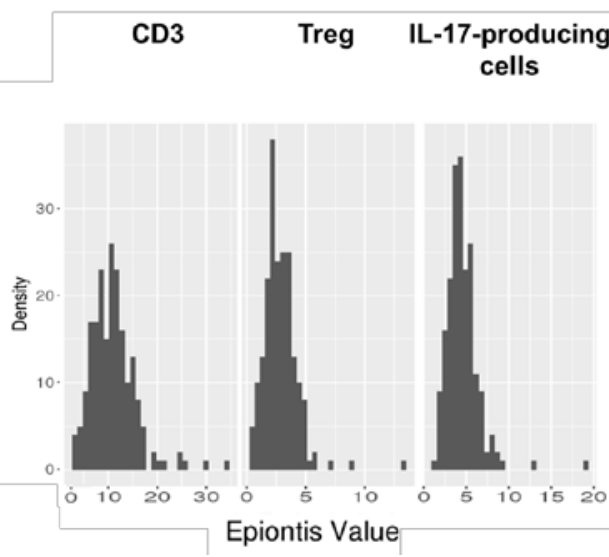

- Frequencies of Treg and IL-17 producing cells at baseline were similar to those described in the

Baseline Cell Frequencies Do Not Correlate With Baseline PASI Scores

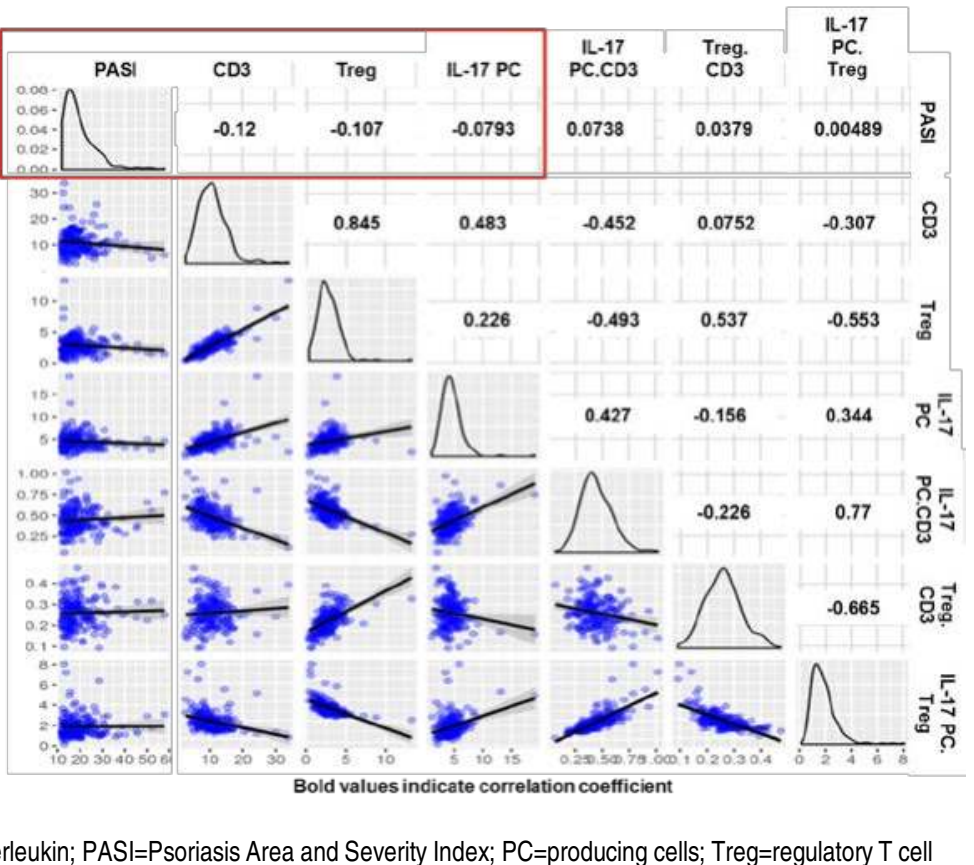

Mirikizumab Decreases Frequency of Resident Skin Immune Cells

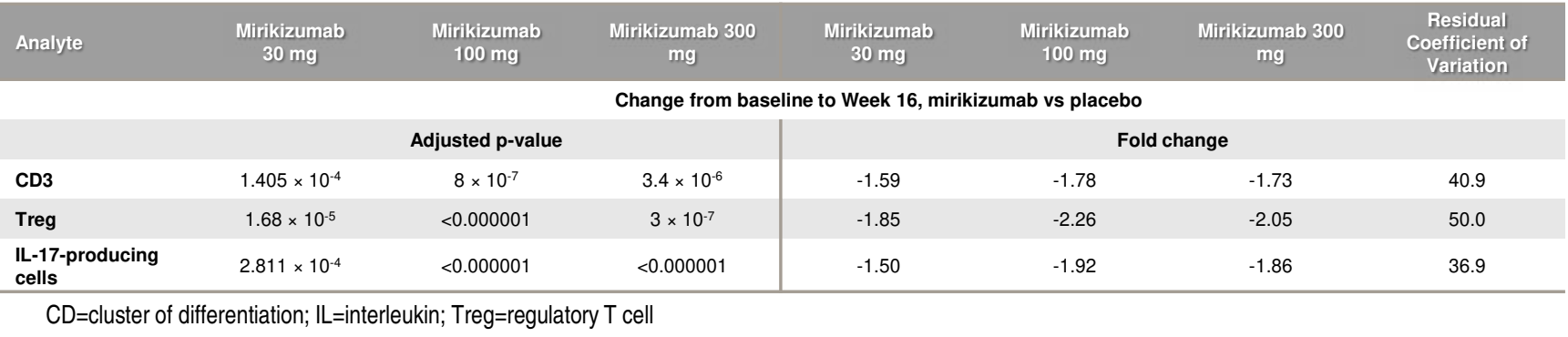

Regardless of dose, mirikizumab significantly reduced the number of immune cell subsets compared to placebo

\section{DISCUSSION}

- Mirikizumab decreased T cell frequency within psoriasis skin

- IL-17-producing cells, specifically, showed a reduction that inversely correlated with clinical response

- Mirikizumab may, through its unique mechanism of action, create a different skin milieu after treatment in psoriatic skin than anti-IL-17 and anti-TNF treatments

\section{LIMITATIONS}

- Exploratory

- Single, small ( $\mathrm{n}$ 50/arm) phase 2 study

- Correlation with clinical data (flare frequencies after last dose at Week 16) not done

\section{CONCLUSIONS}

- In an exploratory assessment, mirikizumab's inhibition of IL-23 reduced the total number of IL-17-producing cells

- This may lead to a more stable response with longer periods of skin clearance without flares

- To further understand the mechanism of action of mirikizumab, future translational trials are needed Dr. Bissonnette has served as a speaker, advisor, investigator, and/or Ingelheim, Celgene, Eli Lilly and Company, Galderma, GSK, Steifel, Incyte Janssen, Leo Phat
Bausch Health

Drs. Schmitz, Patel, Higgs, Sonnergren, and Liu are current employees and Shareholders of Eli Lilly and Company

Dr. Reich has served as advisor and/or paid speaker for and/or participated in clinical trials sponsored by AbbVie, Amgen, Biogen, Boehringer Ingelheim, Celgene, Covagen, Forward Pharma, GlaxoSmithKline, Janssen-Cilag, LEO Pharma, Lilly, Medac, Merck Sharp \& Dohme, Novartis, Pfizer, Regeneron,
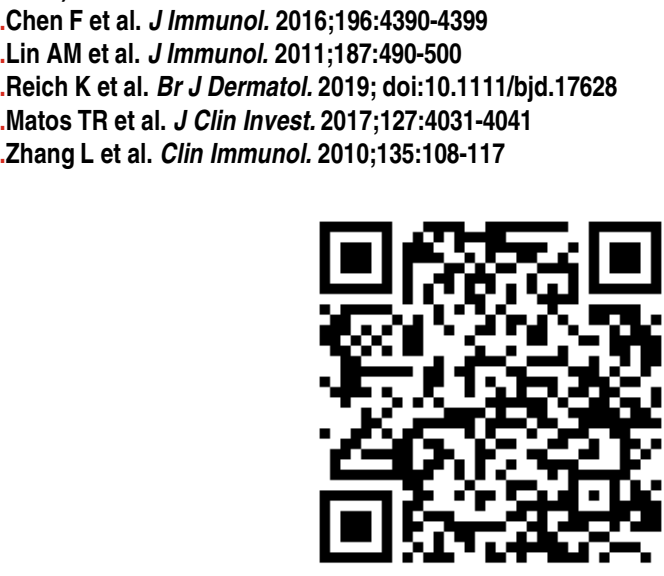